\title{
A semigroup is a rectangular band if and only if it is nowhere commutative
}

\author{
Open Mathematics Collaboration*广
}

July 12, 2020

\begin{abstract}
We prove the proposition addressed in the title of this paper.
\end{abstract}

keywords: semigroup, rectangular band, nowhere commutative, abstract algebra

The most updated version of this paper is available at https://osf.io/98khs/download

\section{Theorem}

1. A semigroup $\mathcal{S}$ is a rectangular band if and only if

$$
\forall a, b \in \mathcal{S}:(a b=b a) \rightarrow(a=b)
$$

2. $[1,2]$

*All authors with their affiliations appear at the end of this paper.

†Corresponding author: mplobo@uft.edu.br | Join the Open Mathematics Collaboration 


\section{Notation \& Definition}

$3 . \cong$ isomorphism

4. $\equiv$ equivalent

5. $\exists$ ! exists exactly one

6. $\mathcal{S}=$ semigroup

7. $L=$ left zero semigroup; $R=$ right zero semigroup

8. $(\forall x, y \in \mathcal{S}: x y=x) \equiv(\mathcal{S}=$ left zero semigroup $)$

9. $(\forall x, y \in \mathcal{S}: x y=y) \equiv(\mathcal{S}=$ right zero semigroup $)$

10. $(\mathcal{S}=$ rectangular band $) \equiv$

$\equiv\left(\left(\forall x \in \mathcal{S}: x^{2}=x\right) \wedge(\forall a, b, c \in \mathcal{S}: a b c=a c)\right)$

$\equiv(\exists L, R: \mathcal{S} \cong L \times R)$

$\equiv\left((\mathcal{S} \cong A \times B) \wedge(A, B \neq \varnothing) \wedge\left(\left(a_{1}, b_{1}\right)\left(a_{2}, b_{2}\right)=\left(a_{1}, b_{2}\right)\right)\right)$

11. $(\forall a, b \in \mathcal{S}:(a b=b a) \rightarrow(a=b)) \equiv$ (nowhere commutative)

\section{To be proved}

12. $(\mathcal{S}=$ rectangular band $) \leftrightarrow(\forall a, b \in \mathcal{S}:(a b=b a) \rightarrow(a=b))$

\section{Proof}

13. $(\rightarrow)$

14. Suppose $\mathcal{S}$ is a rectangular band.

15. According to (14), $\mathcal{S}$ satisfies all equivalences in (10).

16. So we have

$$
\left(\forall x \in \mathcal{S}: x^{2}=x\right) \wedge(\forall a, b, c \in \mathcal{S}: a b c=a c) .
$$


17. Suppose $a, b \in \mathcal{S}$ arbitrary such that $a b=b a$.

18. Using (16) and (17),

$$
(a b=b a) \rightarrow\left(b a b=b^{2} a\right) \rightarrow(b a b=b a) \rightarrow(b b=b a) \rightarrow(b=b a) .
$$

19. Again, using (16) and (17),

$$
(a b=b a) \rightarrow\left(a^{2} b=a b a\right) \rightarrow(a b=a a) \rightarrow(a b=a) \rightarrow(a=b a) .
$$

20. (18) and (19) leads to $a=b$.

21. From (17) and (20), $\forall a, b \in \mathcal{S}:(a b=b a) \rightarrow(a=b)$.

22. From (14) and (21),

$(\mathcal{S}=$ rectangular band $) \rightarrow(\forall a, b \in \mathcal{S}:(a b=b a) \rightarrow(a=b))$.

23. $(\leftarrow)$

24. Suppose $\forall a, b \in \mathcal{S}:(a b=b a) \rightarrow(a=b)$.

25 . Let $a, b \in \mathcal{S}$ arbitrary.

26. Since $\mathcal{S}$ is associative, $a(a a)=(a a) a$.

27. Since $a$ and $a^{2}$ commute, from (24), $a=a^{2}$.

28. Using associativity and idempotency,

$$
a(a b a)=a a b a=a b a=a b a a=(a b a) a .
$$

29. $a$ and $a b a$ commute, so $a=a b a$.

30. For all $c \in \mathcal{S}$, using associativity and (29),

$$
(a c)(a b c)=(a c a)(b c)=a(b c)=(a b)(c a c)=(a b c)(a c) .
$$

31. Since $a c$ and $a b c$ commute, $a c=a b c$.

32. Thus, from (10), (24), (27) and (31), $(\forall a, b \in \mathcal{S}:(a b=b a) \rightarrow(a=b)) \rightarrow(\mathcal{S}=$ rectangular band $)$. 
33. Therefore, from (22) and (32),

$$
(\mathcal{S}=\text { rectangular band }) \leftrightarrow(\forall a, b \in \mathcal{S}:(a b=b a) \rightarrow(a=b)) \text {. }
$$

\section{Open Invitation}

Review, add content, and co-author this paper $[3,4]$. Join the Open Mathematics Collaboration (https://bit.ly/ojmp-slack). Send your contribution to mplobo@uft.edu.br.

\section{Open Science}

The latex file for this paper together with other supplementary files are available [5].

\section{Ethical conduct of research}

This original work was pre-registered under the OSF Preprints [6], please cite it accordingly [7]. This will ensure that researches are conducted with integrity and intellectual honesty at all times and by all means.

\section{Acknowledgement}

+ Center for Open Science https://www.cos.io

+ Open Science Framework https://osf.io 


\section{References}

[1] Howie, John Mackintosh. Fundamentals of semigroup theory. Oxford University Press, 1995.

[2] Cain, Alan J. Nine Chapters on the Semigroup Art. AJC Porto \& Lisbon, 2020. http://www-groups.mes.standrews.ac.uk/\%7ealanc/teaching/m431/

[3] Lobo, Matheus P. "Microarticles." OSF Preprints, 28 Oct. 2019. https://doi.org/10.31219/osf.io/ejrct

[4] Lobo, Matheus P. "Simple Guidelines for Authors: Open Journal of Mathematics and Physics." OSF Preprints, 15 Nov. 2019. https://doi.org/10.31219/osf.io/fk836

[5] Lobo, Matheus P. "Open Journal of Mathematics and Physics (OJMP)." OSF, 21 Apr. 2020. https://doi.org/10.17605/osf.io/6hzyp

[6] COS. Open Science Framework. https://osf.io

[7] Lobo, Matheus P. "A Semigroup Is a Rectangular Band If and Only If It Is Nowhere Commutative." OSF Preprints, 7 July 2020. https://doi.org/10.31219/osf.io/98khs

\section{The Open Mathematics Collaboration}

Matheus Pereira Lobo (lead author, mplobo@uft.edu.br) $)^{1,2}$ https://orcid.org/0000-0003-4554-1372

José Carlos de Oliveira Junior ${ }^{1}$ https://orcid.org/0000-0002-6518-5566

${ }^{1}$ Federal University of Tocantins (Brazil)

${ }^{2}$ Universidade Aberta (UAb, Portugal) 